\title{
Role of cardiovascular magnetic resonance in the evaluation of cardiomyopathy
}

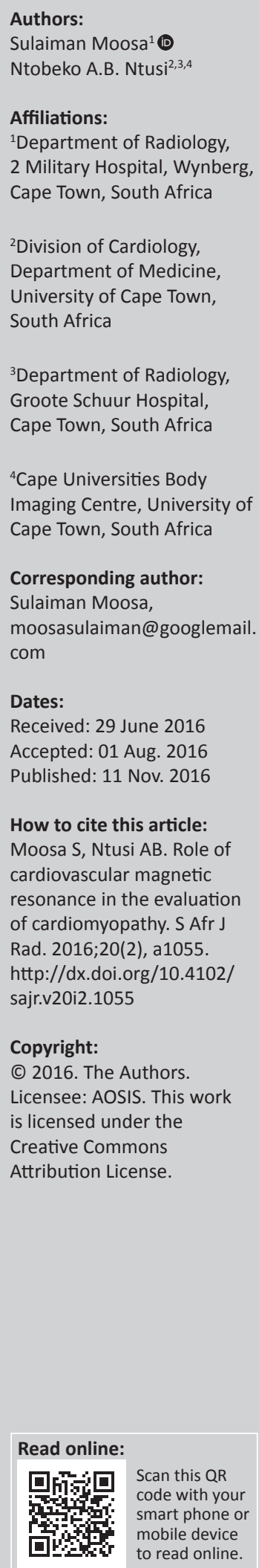

Cardiovascular magnetic resonance imaging plays a central role in the assessment and monitoring of patients with cardiomyopathy. It offers a comprehensive assessment during a single scan setting, with information on ventricular volumes, function and mass as well as tissue characterisation, fibrosis, flow, viability and perfusion. Acute tissue injury (oedema and necrosis) can be distinguished from fibrosis, infiltration and iron overload. It provides information on the cause and prognosis of the cardiomyopathy, and its high measurement accuracy makes it ideal for monitoring disease progression and effects of therapy. The present review highlights the main features of commonly encountered cardiomyopathies in imaging practice.

\section{Introduction}

Cardiomyopathies comprise a wide range of myocardial disorders that may be primary heart diseases or a feature of a systemic disease, and which may or may not have a genetic and/or familial component. ${ }^{1}$ Cardiomyopathy is defined as a:

\begin{abstract}
... myocardial disorder in which the heart muscle is structurally and functionally abnormal, in the absence of coronary artery disease, hypertension, valvular disease and congenital heart disease sufficient to cause the observed myocardial abnormality. ${ }^{2}$
\end{abstract}

The key aim in the work-up of suspected cardiomyopathy is to identify a potentially treatable underlying cause, to risk-stratify the patient for prognostication, and to apply an effective therapeutic strategy. Besides evidence-based pharmacological therapeutic options, therapy includes consideration for an implantable cardioverter defibrillator (ICD), cardiac resynchronisation and orthotopic heart transplant. ${ }^{1}$

Cardiovascular magnetic resonance (CMR) provides the opportunity to obtain key information during a single non-invasive study. Data on cardiac anatomy, function, tissue characterisation, coronary and microvascular perfusion and valvular structure and function in any selected orthogonal plane, independent of the patient habitus, can be safely acquired. The absence of ionising radiation adds to its safety. This safety feature includes intravenous contrast which allows repeated follow-up imaging, family screening and serial risk stratification.

\section{Technical aspects and sequences}

A typical cardiomyopathy protocol is as follows, but should be adjusted as dictated by the clinical suspicion and continuous assessment of images during the scan.

\section{Gross anatomical images}

Half-Fourier single-shot turbo spin-echo (HASTE) in two to three orthogonal planes.

\section{Functional imaging}

Balanced steady state free precession (b-SSFP) in long-axis and short-axis views forms the cornerstone of CMR, for reproducible quantification of LV (left ventricle) and RV (right ventricle) function and LV mass.

\section{Non-contrast tissue characterisation}

- T2-weighted and STIR images - oedema detection

- T1-weighted images - pericardium and fat infiltration assessment

- T2* images - direct quantification of myocardial iron in vivo. 


\section{Imaging fibrosis (scar) and infiltration}

Gadolinium-based contrast agents are utilised. Gadolinium is an extracellular agent that accumulates in regions of interstitial expansion, such as in oedema, fibrosis or infiltration. After an intravenous gadolinium injection (usual dose $0.1 \mathrm{mmol} / \mathrm{kg}-0.2 \mathrm{mmol} / \mathrm{kg}$ of body mass), three postcontrast phases can be assessed at the following times:

- First pass is immediate imaging at rest or during stress perfusion to visualise inducible perfusion defects.

- Early gadolinium enhancement (EGE), acquired at $90 \mathrm{~s}-$ $120 \mathrm{~s}$, detects thrombi, hyperaemia and microvascular obstruction post myocardial infarction.

- Late gadolinium enhancement (LGE) acquired at 5 min $20 \mathrm{~min}$ detects delayed contrast washout in areas of infarction, fibrosis or inflammation.

Sequences utilised for LGE include inversion recovery gradient echo (magnitude images) or phase sensitive inversion recovery (PSIR) images, the latter being less dependent on accurate inversion time, but which is an absolute prerequisite for the former. ${ }^{1,3}$

LGE demonstrates fibrosis (scar) by revealing the relative difference between the scar (delayed contrast washout) and normal myocardium (rapid washout). The pattern and extent of LGE varies according to the pathological process and contributes to the correct diagnosis in cardiomyopathy. ${ }^{1}$ Fibrosis is assessed visually, and the extent can be quantified as a percentage of mass using dedicated software.

\section{Myocardial mapping}

More recently, myocardial mapping techniques have expanded our ability to characterise myocardium without contrast administration. T1 mapping measures the absolute myocardial $\mathrm{T} 1$ relaxation time on a pixel-by-pixel basis, hence being able to detect subtle changes in T1 relaxation times, the latter being proportional to the local concentration of gadolinium. Fibrosis causes expansion of the interstitial space and results in increased native $\mathrm{T} 1$ times, and the increased gadolinium concentration results in shortening of T1 values. ${ }^{1}$ T1 mapping is also useful for detecting small amounts of fibrosis and cardiomyopathies with diffuse fibrosis. ${ }^{4}$ Recently, T2 mapping ${ }^{5}$ and T2* mapping ${ }^{6}$ has been validated for imaging of myocardial oedema and/or inflammation and iron content, respectively.

\section{Flow quantification}

CMR velocity mapping can provide reproducible assessment of valvular disease, intracardiac shunts and congenital heart disease, and compares well with echocardiography. ${ }^{7}$

\section{Limitations and contraindications}

Metallic implants and intracardiac devices (pacemakers and ICDs) represent the only absolute contraindications to CMR. However, this is being overcome with the increasing use of MR-compatible devices. Metallic objects in delicate positions (e.g. within the eye) continue to pose a problem as ferromagnetic objects may act as projectiles within the powerful magnetic field of the scanner and cause injury. Relative contraindications include claustrophobia, florid heart failure and clinical instability. CMR up to 3 Telsa is safe during all trimesters of pregnancy. ${ }^{8}$

Severe renal failure (glomerular filtration rate $[\mathrm{GFR}]<30 \mathrm{~mL}$ / min) precludes the use of gadolinium owing to a small but tangible risk of nephrogenic systemic fibrosis, which is enhanced by concomitant heart failure (HF). ${ }^{1}$ The limited availability of CMR scanners and CMR expertise are currently the most important limitations.

\section{A practical approach to an unknown cardiomyopathy}

The imaging clinician's first task is to obtain maximum information about the suspected diagnosis by evaluating clinical notes, operative notes, and previous investigations such as electrocardiograms (ECGs), echocardiography (echo), cardiac tomography and prior CMR studies.

After performing the anatomical and functional sequences to assess heart morphology and function, the following features should be identified to assist in optimising scan planning:"

- Myocardial thinning: Global myocardial thinning is often found in dilated cardiomyopathy (DCM), left ventricular noncompaction (LVNC), and arrythmogenic right ventricular cardiomyopathy (ARVC). Regional thinning suggests chronic myocardial infarction, sarcoidosis and burnt-out hypertrophic cardiomyopathy (HCM).

- Enlarged RV: A dilated RV indicates ARVC, DCM, sarcoid, chronic myocarditis, intracardiac shunt (e.g. atrial septal defect) and congenital disease (e.g. tetralogy of Fallot, Ebstein's anomaly).

- Myocardial hypertrophy: Increased myocardial thickness points to HCM, cardiac amyloidosis, Anderson-Fabry disease and cardiomyopathy related to mitochondrial disease. However, pressure overload conditions such as hypertension, aortic stenosis and aortic coarctation should always be considered.

- Biatrial enlargement: Marked atrial enlargement indicates a restrictive cardiomyopathy, amyloidosis, constrictive pericarditis and DCM.

\section{Indications for CMR}

CMR is considered as a Class I indication for global and regional LV and RV function, volume and mass quantification. ${ }^{10,11}$ Indications include:

- systolic function assessment in patients with poor echocardiographic acoustic window and precise ejection fraction (EF) determination that will affect management or device implantation,

- prognostic information from the LGE burden,

- reproducible LV mass assessment and follow-up,

- RV evaluation in suspected cardiomyopathies, right ventricular ejection fraction (RVEF), wall motion abnormalities, oedema and scarring, 
- $\quad$ assessing the cause of LV dysfunction in newly diagnosed heart failure; it can play a role as an effective gatekeeper to coronary angiograms in such patients,

- screening family members with cardiomyopathy,

- screening athletes with syncope or aborted sudden cardiac death (SCD),

- chest pain syndrome (CPS) with unobstructed coronaries,

- suspected myocarditis, congenital heart disease and infiltrating conditions.

\section{Dilated cardiomyopathy}

DCM refers to LV systolic dysfunction with concomitant LV dilation; RV dilatation may be present. In $50 \%$ of cases, the diagnosis remains unknown; up to $30 \%$ have a familial association to known genetic abnormalities. ${ }^{12}$ Chronic myocarditis has been implicated in the aetiology of DCM. $^{12}$

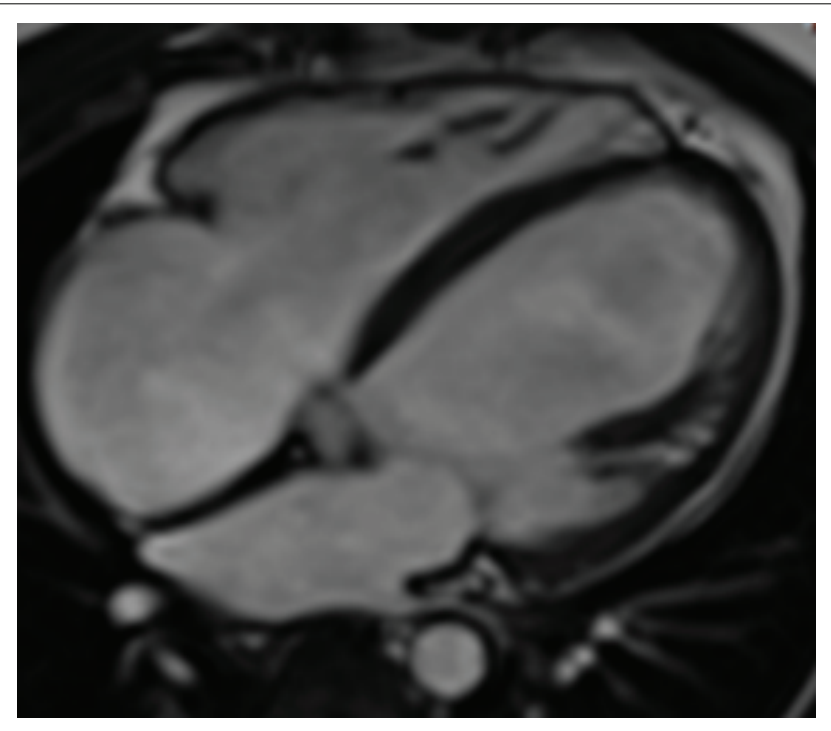

FIGURE 1: Dilated cardiomyopathy. Cine b-SSFP in end-diastole demonstrates enlarged LV volumes with the end-diastolic diameter $67 \mathrm{~mm}$. The end-systolic diameter was $58 \mathrm{~mm}$.
CMR provides accurate evaluation of biventricular EFs, volumes and mass assessment, and the pattern of LGE allows the exclusion of an ischaemic cause, typically subendocardial or transmural enhancement. LGE provides prognostic information and acts as a gatekeeper role to coronary angiography. ${ }^{13}$

In a large study with DCM and unobstructed coronary arteries, three LGE patterns were reported: no LGE in 59\%, midwall and mainly septal LGE in $28 \%$, and subendocardial ischaemic LGE pattern in $13 \%$, probably on the basis of transient occlusion by non-obstructive unstable plaque or vasoconstriction. ${ }^{13}$

Other studies have shown additional LGE patterns that include epicardial LGE, patchy LGE and diffuse LGE. ${ }^{14}$ The finding of LGE in DCM is associated with increased risk of SCD, presumably arrhythmogenic.

Figure 1 and Figure 2 provide the representative imaging features of DCM.

\section{Left ventricular noncompaction}

LVNC is a rare congenital cardiac disorder owing to arrested embryogenesis and results in a 'spongy' appearance of the LV myocardium and apex, commonly involving the anterior and lateral walls and characterised by increased trabeculation and intratrabecular recesses in the myocardium. ${ }^{16}$ LVNC is associated with neuromuscular disorders and is complicated by heart failure, arrhythmia and thromboembolic events. ${ }^{1}$ Diagnosis is achieved by measuring at end-diastole, the ratio of the noncompact myocardium over the compact myocardium. A value of 2.3 has a sensitivity of $80 \%$ and specificity of $99 \%{ }^{17}$

The absence of well-formed papillary muscles is a clue to the diagnosis. ${ }^{1}$ LGE patterns include subendocardial, midwall and transmural enhancement. ${ }^{1}$ LVNC is commonly associated with a DCM phenotype. Figure 3 demonstrates the imaging features of LVNC.
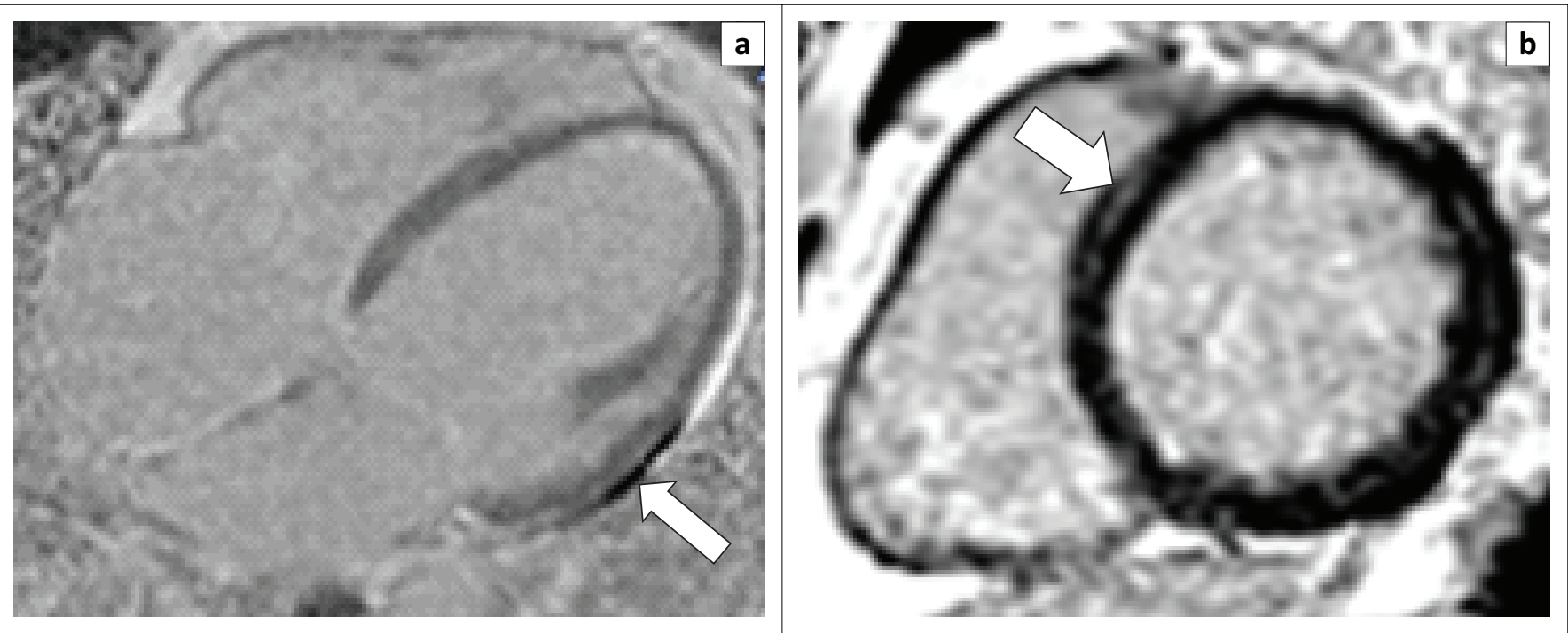

FIGURE 2: Dilated cardiomyopathy. Late gadolinium-enhanced PSIR images in four-chamber (left) and basal short-axis (right) demonstrating virtual circumferential midwal enhancement (arrows). 


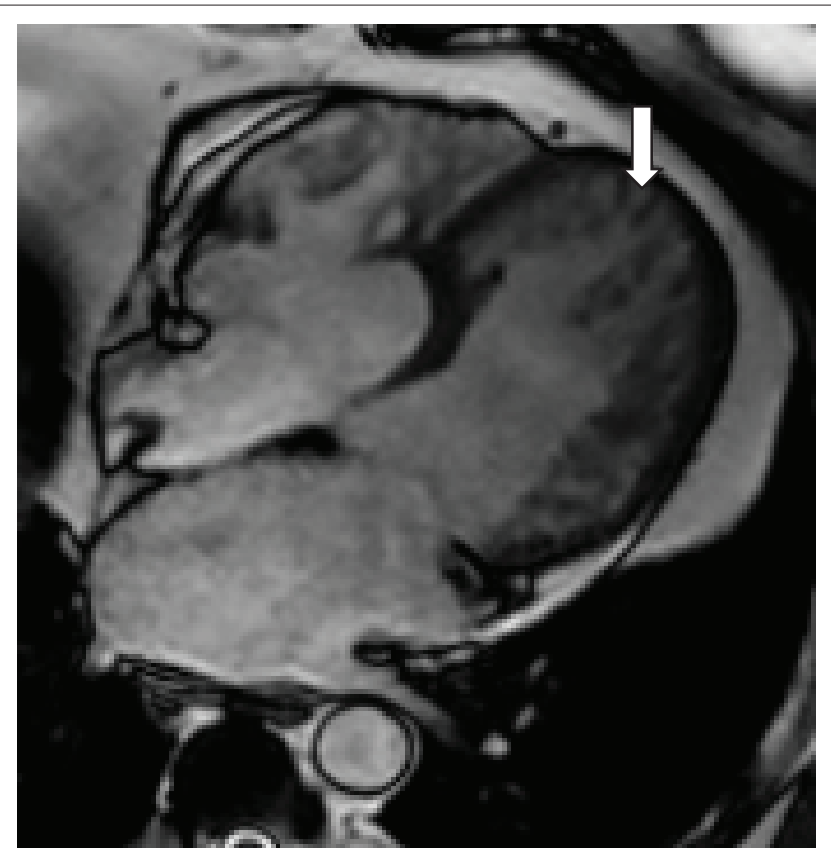

FIGURE 3: Left ventricular noncompaction. Four-chamber cine b-SSFP demonstrating increased LV trabeculations (arrow) at the LV free wall and apex compared with the compact LV myocardium. The RV trabeculations are prominent too.

\section{Arrhythmogenic right ventricular cardiomyopathy}

ARVC is a genetic desmosomal disease characterised pathologically by progressive RV myocyte loss and fibrofatty replacement. ${ }^{1}$ In $75 \%$ of cases, the LV is affected and hence it can be misdiagnosed as DCM, especially in phenotypes with early and predominant LV involvement. ${ }^{1}$ ARVC should be considered in all cases of unexplained RV dilation. Aborted SCD from arrhythmia is often the initial presentation.

ARVC task force criteria (TFC) forms the cornerstone of the diagnosis, and the 2010 revision has improved sensitivity for gene carriers with limited disease and patients with leftsided disease. ${ }^{18}$ The values for RVEF and EDV have been defined on the basis of comparison with normal patient data and divided into two categories.

\section{Major criteria}

RV regional wall motion abnormality (akinesia, dyssynchrony or aneurysm) and one of the following:

- RVEF $\leq 40 \%$

- $\mathrm{RV}$ end-diastolic volume $\geq 110 \mathrm{~mL} / \mathrm{m}^{2}$ for male and $\geq 100 \mathrm{~mL} / \mathrm{m}^{2}$ for female patients.

\section{Minor criteria}

Regional wall motion abnormality and one of the following:

- $\quad$ RVEF $>40 \%$ and $\leq 45 \%$

- $\mathrm{RV}$ end-diastolic volume $\geq 100 \mathrm{~mL} / \mathrm{m}^{2}-<110 \mathrm{~mL} / \mathrm{m}^{2}$ for male and $\geq 90 \mathrm{~mL} / \mathrm{m}^{2}-<100 \mathrm{~mL} / \mathrm{m}^{2}$ for female patients.

Taking into account the revised TFC for the diagnosis of ARVC, a definite diagnosis of ARVC is made when a patient has:
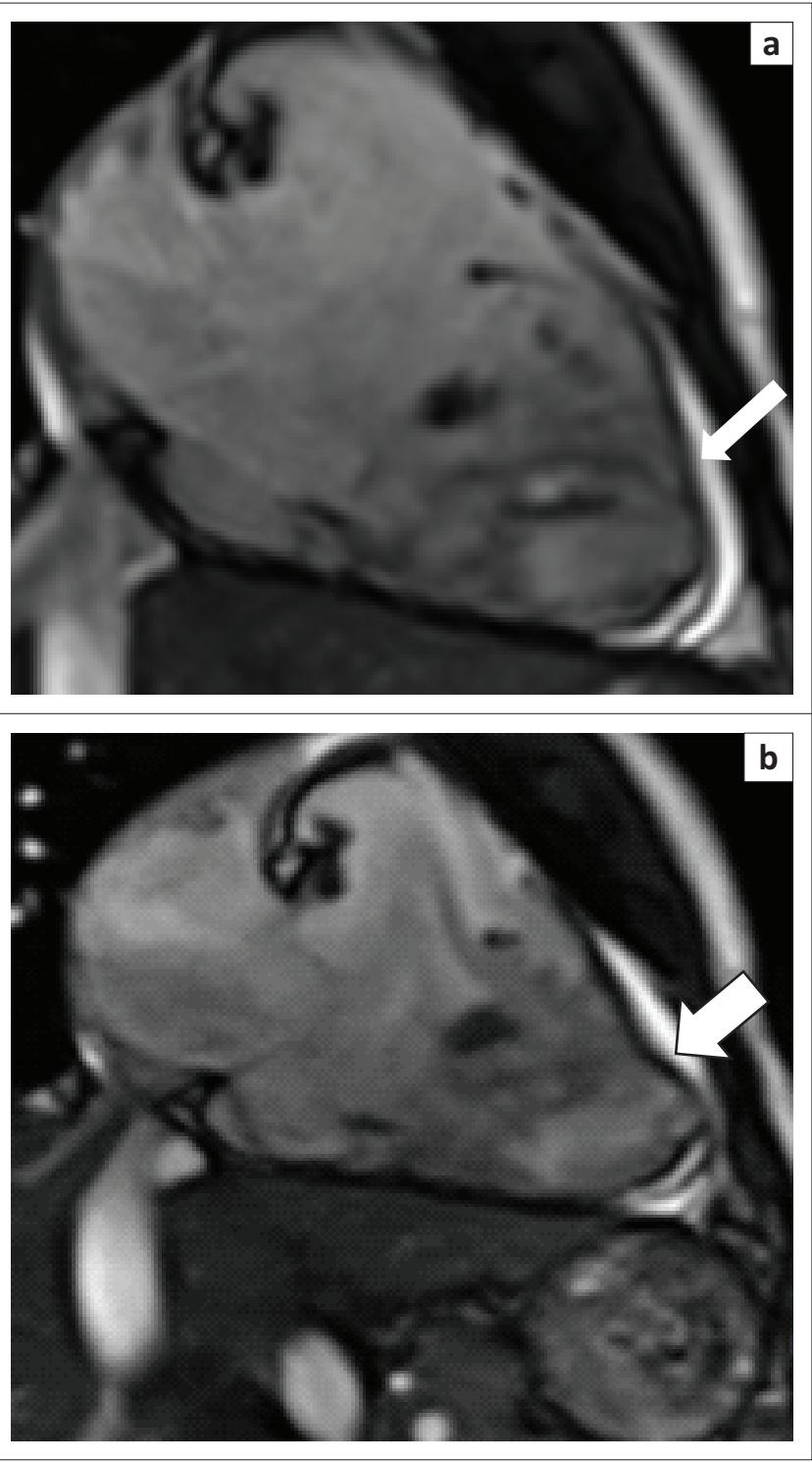

FIGURE 4: Arrhythmogenic right ventricle cardiomyopathy. RV two-chamber views cine b-SSFP in end-diastole (left) and end-systole (right) demonstrating a dyskinetic RV apex (arrows)

- two major criteria

- one major and two minor criteria

- four minor criteria. ${ }^{9}$

A borderline diagnosis of ARVC is made when a patient has:

- one major and one minor criterion

- three minor criteria.

A possible diagnosis of ARVC is made when a patient has:

- one major or two minor criteria.

Major and minor criteria have been defined for histology, ECG and family history, and are applied in the diagnosis. ${ }^{18}$

CMR transaxial cine stack is included as part of the work-up and detects regional and global RV dysfunction, wall motion abnormalities, aneurysms and wall thinning. 
T1-weighted fat detection and LGE are not part of the revised ARVC TFC. Fat can appear in the RV in healthy individuals and can be difficult to image in the thin RV wall or discriminate with confidence from epicardial fat. LGE is part of the ARVC protocol and its absence does not preclude the diagnosis but, when present, it has a high diagnostic sensitivity and specificity. ${ }^{19}$

However, RV LGE presents imaging problems: ${ }^{20}$ Firstly, it is difficult to discriminate from RV fat; and secondly it requires significantly different inversion times compared with the LV. However, LGE patterns of the RV can delineate different patterns of ARVC: (1) classic form - LGE in inferior and inferolateral walls; and (2) left-predominant phenotype septal midwall enhancement with preserved RV function. This type is often misdiagnosed as DCM. Figure 4 demonstrates RV free wall dyskinesia, typical of ARVC.

\section{Hypertrophic cardiomyopathy}

HCM is the most common genetic cardiomyopathy, with an incidence of 1 in 500. It is also the most common cause of sudden death in the young, including athletes. Clinical findings and abnormal ECG findings associated with apical or asymmetrical septal hypertrophy, with or without dynamic LV outflow tract obstruction, is diagnostic. Pathologically, HCM demonstrates myocyte hypertrophy, disarray and fibrosis, manifesting in various genetic mutations. ${ }^{1}$ The majority of individuals carrying an HCM genetic defect do not manifest the clinical expression at all times of their lives. Hence an unexplained mildly thickened LV wall may be consistent with a HCM-causing mutation. Making a diagnosis of HCM can be complicated in patients with aortic stenosis or hypertension. ${ }^{1}$

Cine images allow the detection of LV wall thickness, especially at the apex, basal anteroseptal wall and posterolateral wall - regions that are difficult to visualise on echocardiography. However, a spectrum of LV wall thickening has been described. ${ }^{21}$ Myocardial crypts are additional typical findings. Apical aneurysms are associated with an increased risk for arrhythmia, thromboembolic events and progressive heart failure. ${ }^{22}$

LGE is present in $80 \%$ of HCM, both patchy or diffuse scarring, the latter in areas of increased thickness typically and less commonly in regions of normal wall thickness. ${ }^{23}$ Patchy LGE is typically midwall in thickened myocardium and commonly the RV-LV insertion points are also involved. In burnt-out HCM, the myocardium is thinned and has transmural LGE, mimicking ischaemic disease, but not in a coronary artery territory.

HCM without LGE has 100\% event-free survival at 6-year follow-up; LGE > 5\% of LV mass, septal wall thickness > 30 $\mathrm{mm}$ and AF are independent predictors of death and ICD discharges. ${ }^{24}$

Left ventricular outflow tract (LVOT) obstruction and the presence of systolic anterior motion of the mitral valve can be quantified with velocity flow mapping and has been correlated with increased severity of hypertrophy, heart failure and poor outcome. ${ }^{25}$

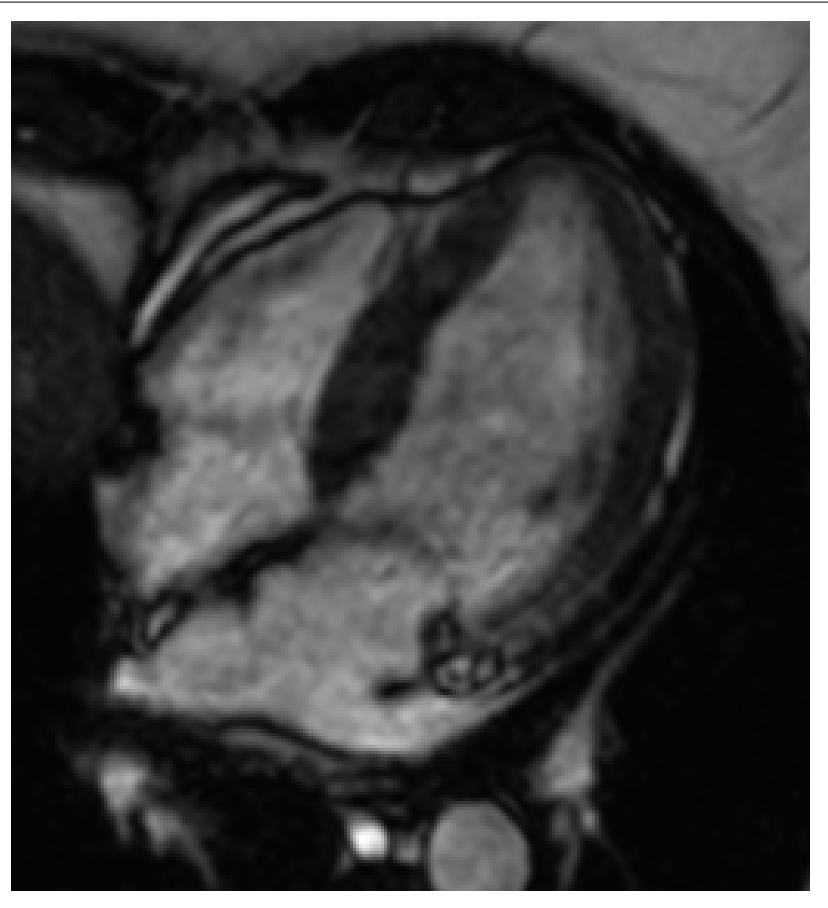

FIGURE 5: Hypertrophic cardiomyopathy. Four-chamber end-diastole cine b-SSFP image demonstrating a thickened septum and LV lateral wall.

Figure 5 and Figure 6 demonstrate the imaging features of HCM.

\section{Amyloidosis}

Myocardial involvement in systemic amyloidosis is an important prognostic marker, often presenting as congestive heart failure in $50 \%$ of patients, and death occurring in less than 6 months in untreated patients. Early detected cases may respond to therapy, and exclusion of other causes is important for management. ${ }^{26}$ Typical CMR findings include normal or reduced LV function, small LV cavity with homogeneous wall thickening, inconsistent RV involvement, biatrial enlargement, interatrial wall thickening, valve thickening, small pericardial effusions and pleural effusions. About 55\% of patients with amyloidosis present with asymmetrical septal hypertrophy mimicking HCM. ${ }^{1}$

LGE reflects the distribution of amyloid on histology with widespread subendocardial enhancement, giving the typical 'zebra' pattern and sparing the midwall of the septum. Other patterns are midwall (33\%), and subepicardial (up to $20 \%){ }^{27}$ RV free wall and interatrial septum LGE has been reported. ${ }^{1}$

When amyloid fibrils accumulate in the myocardium, the wash-in and washout features of gadolinium are abnormal, with inability to null the myocardial signal. The rapid washout from the blood pool and high myocardial uptake results in a dark blood pool, owing to similar T1 values to the visualised myocardium. ${ }^{1}$ To optimally visualise myocardial and blood pool kinetics in suspected amyloidosis, an inversion time scout is acquired at $5 \mathrm{~min}$ after contrast administration. LGE has a sensitivity of $86 \%$ for detecting 


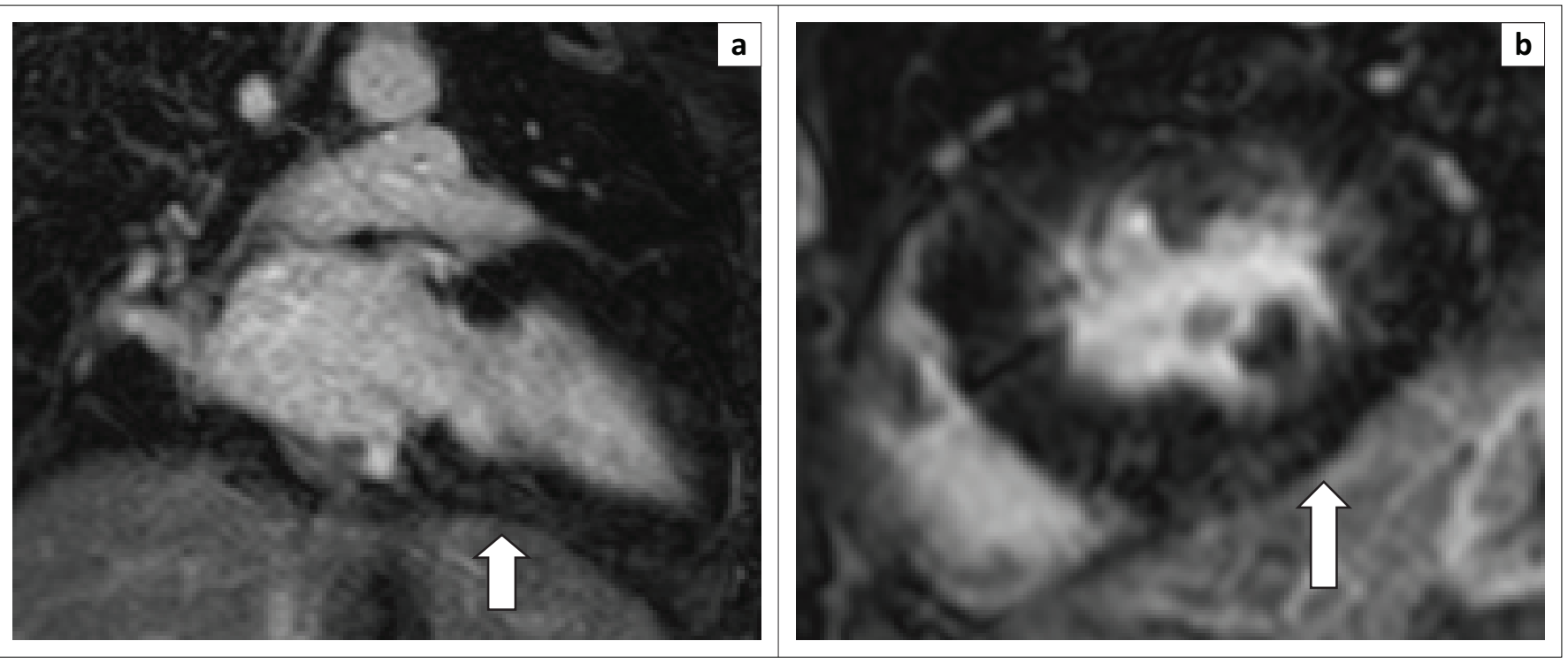

FIGURE 6: Hypertrophic cardiomyopathy. Two-chamber (left) and short-axis (right) late gadolinium images demonstrate diffuse enhancement of the thickened myocardium (arrows).
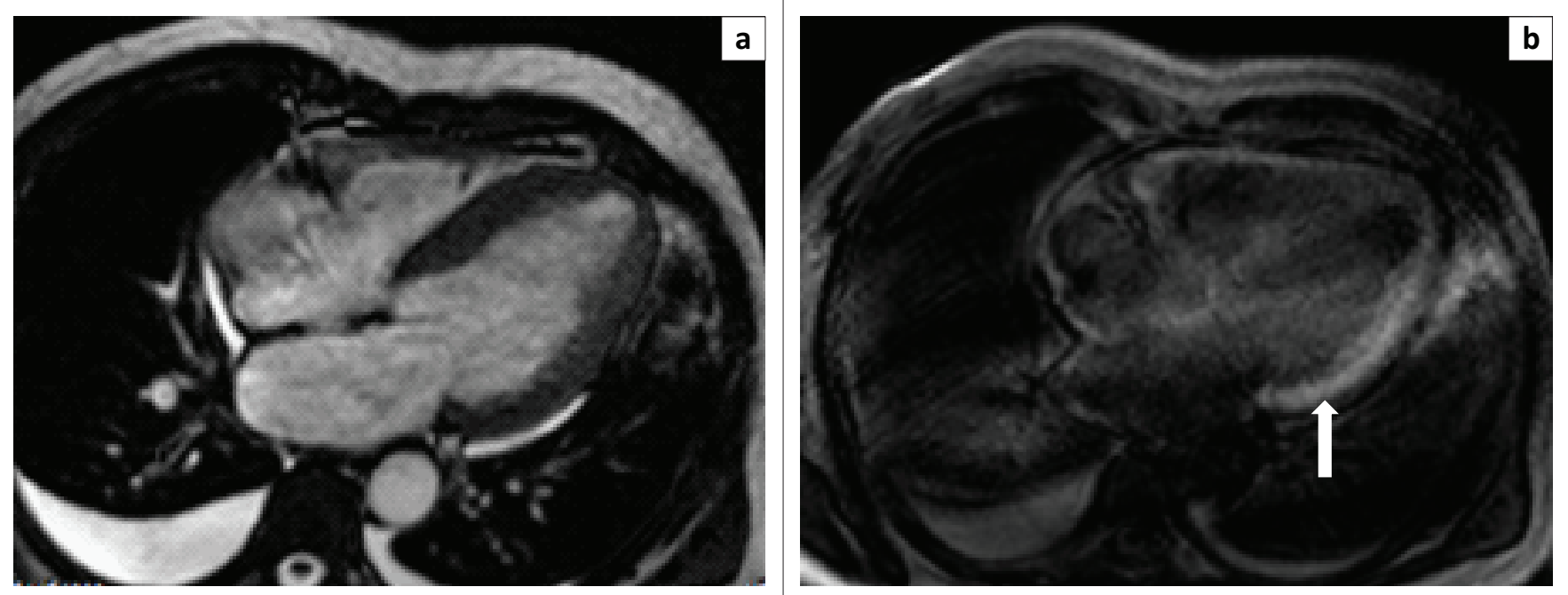

FIGURE 7: Amyloidosis. Four-chamber end-diastole cine b-SSFP image (left) demonstrates a thickened septum and LV lateral wall with small pericardial and pleural effusions. The four-chamber late gadolinium image (right) shows diffuse subendocardial enhancement (arrow) in a non-coronary artery distribution, with enhancement of the RV free wall and interatrial septum.

amyloid. Identifying early abnormalities in patients with normal LV wall thickness is strongly related to the amyloid burden and predicted mortality risk. ${ }^{28,29}$

Figure 7 demonstrates the representative imaging findings of amyloidosis.

\section{Anderson-Fabry disease}

Anderson-Fabry disease is a treatable X-linked disorder of lysosome storage, caused by partial or complete alpha galactosidase A deficiency which results in glycosphingolipid accumulation within the vessels and heart. ${ }^{30}$ Concentric LV hypertrophy and cardiac fibrosis then leads to heart failure. CMR is used to evaluate LV function, wall thickness and scar detection. The typical LGE is inferolateral midwall enhancement which correlates well with histology and may serve as a substrate for electrical re-entry and SCD. ${ }^{31}$ Recently, T1 mapping has been shown to have a relative specific signature for conditions with a decreased T1 value, especially in areas of LGE. ${ }^{32}$ Six per cent of suspected HCM turns out to be Anderson-Fabry, with important implications for management. Anderson-Fabry is treated with enzyme replacement, with resultant improvement in LV function. The enzyme replacement is optimally utilised before fibrosis sets in. An unexplained LV hypertrophy, especially in young patients, should have Anderson-Fabry excluded.

\section{Sarcoidosis}

Sarcoidosis is a multisystem granulomatous disease affecting the myocardium in 50\% of cases, but only half of them are symptomatic. ${ }^{33} \mathrm{HF}$ and arrhythmia are the important presentations, and early identification allows medical therapy and ICD implantation. 


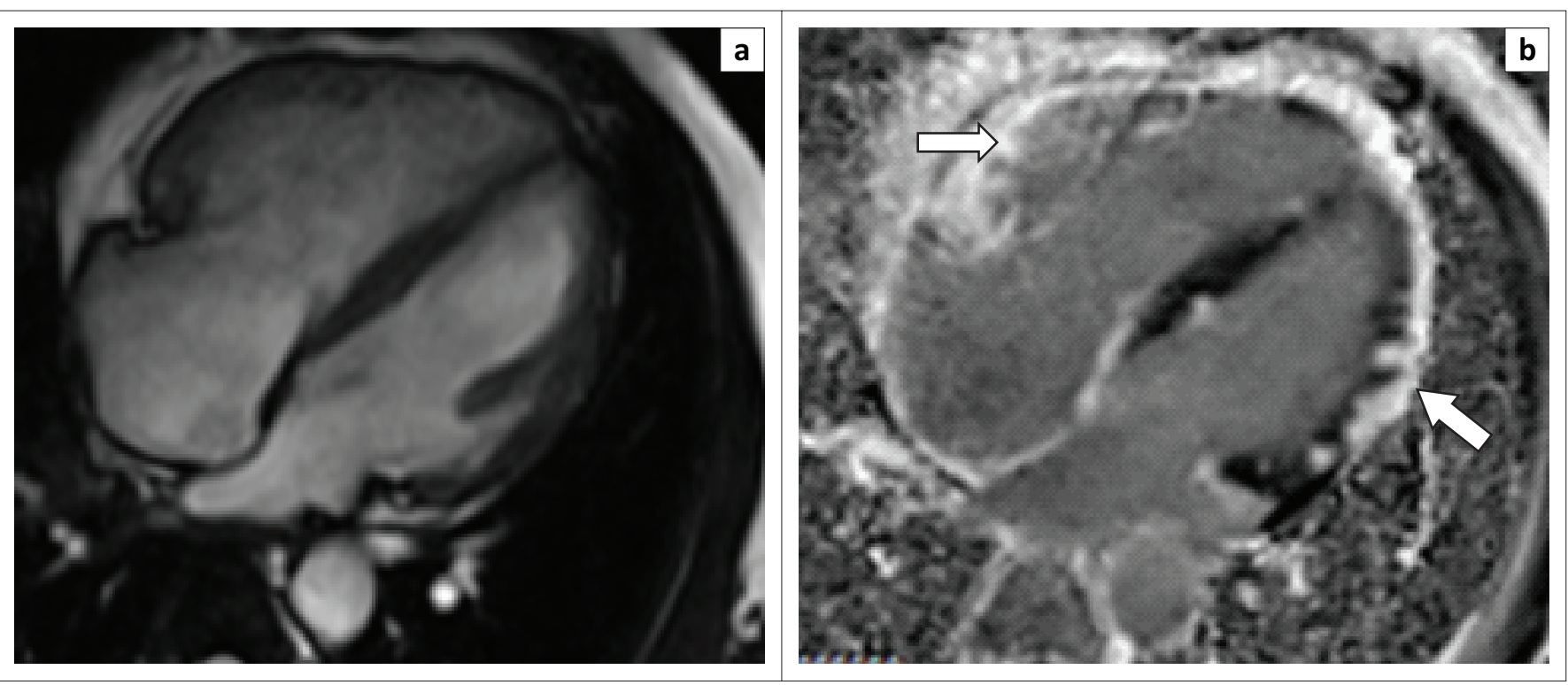

FIGURE 8: Sarcoidosis. End-diastole four-chamber cine b-SSFP image (left) demonstrates increased RV size. The four-chamber late gadolinium PSIR image (right) demonstrates prominent RV free wall enhancement (smaller arrow) as well as nodular enhancement of the septum and LV lateral wall (larger arrow).

CMR cine imaging facilitates the detection of wall thinning, regional wall motion abnormalities, ventricular dysfunction and pericardial effusion, whilst LGE CMR detects fibrosis. LGE is typically patchy, midwall or subepicardial, in an unpredictable manner, in the anteroseptal and anterolateral walls as well as in the RV free wall and RV side of the septum. ${ }^{34}$ Sarcoid may mimic ischaemic disease with subendocardial or transmural patterns. ${ }^{35}$ LGE acts as a guide to endomyocardial biopsy and improves after steroid therapy. T2W imaging may monitor disease activity with inflamed oedematous regions being highlighted. ${ }^{34}$ Recently, T1 and T2 mapping has been shown to have a role in the assessment and monitoring of sarcoidosis. ${ }^{36}$ Figure 8 demonstrates the representative imaging findings of sarcoidosis.

\section{Iron overload cardiomyopathy}

Cardiac siderosis is evident in transfusion-dependent anaemia and primary haemachromatosis. If chelation therapy is initiated early, the cardiomyopathy is reversible; but the diagnosis is often delayed owing to the late onset of symptoms. T2* imaging arises from local magnetic inhomogeneities that increase with greater iron deposition. ${ }^{1}$ As myocardial iron increases, heart failure progresses and T2* times diminish so that all heart failure patients have a T2* value $<20 \mathrm{~m} / \mathrm{sec}$. The efficacy of chelation can also be monitored. ${ }^{1} \mathrm{CMR}$ has a significant role to play in the diagnosis and management of this disease.

\section{Chest pain syndrome with unobstructed arteries}

CMR has increasing utility in providing diagnostic information in patients presenting with troponin-positive CPS with normal coronary arteries. It contributes to a diagnosis in $77 \%$ of patients, correcting a wrong diagnosis in $10 \%$ and changing therapy in $33 \%{ }^{30}$ The main causes of CPS with normal coronary arteries are myocarditis, takotsubo cardiomyopathy, recanalised infarcts and the inflammatory phases of DCM, HCM and ARVC.

\section{Myocarditis}

Myocarditis refers to inflammation of the myocardium that may be caused by infection, autoimmune diseases or drug toxicity; some cases are idiopathic. It has a wide range of clinical manifestations, ranging from an influenza-like viral illness, troponin-positive CPS, new onset HF and even SCD. The diagnosis is usually one of exclusion, and endomyocardial biopsy is the gold standard but sampling bias and insensitivity limits its utilisation; however, CMR-guided biopsies result in higher yields. ${ }^{37}$

CMR is a non-invasive tool to diagnose myocarditis, utilising T2-weighted imaging to detect subendocardial and midwall myocardial oedema. ${ }^{38}$ EGE matching areas of T2-weighted hyperintensity may reflect tissue hyperaemia and increased interstitial space, and LGE reflects myocardial necrosis which has a subepicardial distribution, with varying degrees of midmyocardial involvement; the subendocardium is often spared. The lateral and inferolateral walls are frequently involved. ${ }^{39}$

The presence of a typical LGE pattern is a strong indicator for the diagnosis, but the absence of this pattern does not exclude the diagnosis. ${ }^{40}$ The focal LGE pattern evolves and becomes diffuse over a period of weeks, then decreases during healing and may become invisible after recovery. ${ }^{37}$ Midwall myocardial scarring can be evident after myocarditis, in a pattern similar to that seen in DCM subjects and suggests that previous myocarditis is a cause of LV dysfunction in a proportion of DCM patients. ${ }^{41} \mathrm{~T} 1$ mapping has been shown to be superior to LGE and T2-weighted imaging in the diagnosis of myocarditis, without the need for contrast agent. ${ }^{42}$ Figure 9 demonstrates the representative imaging findings of myocarditis. 


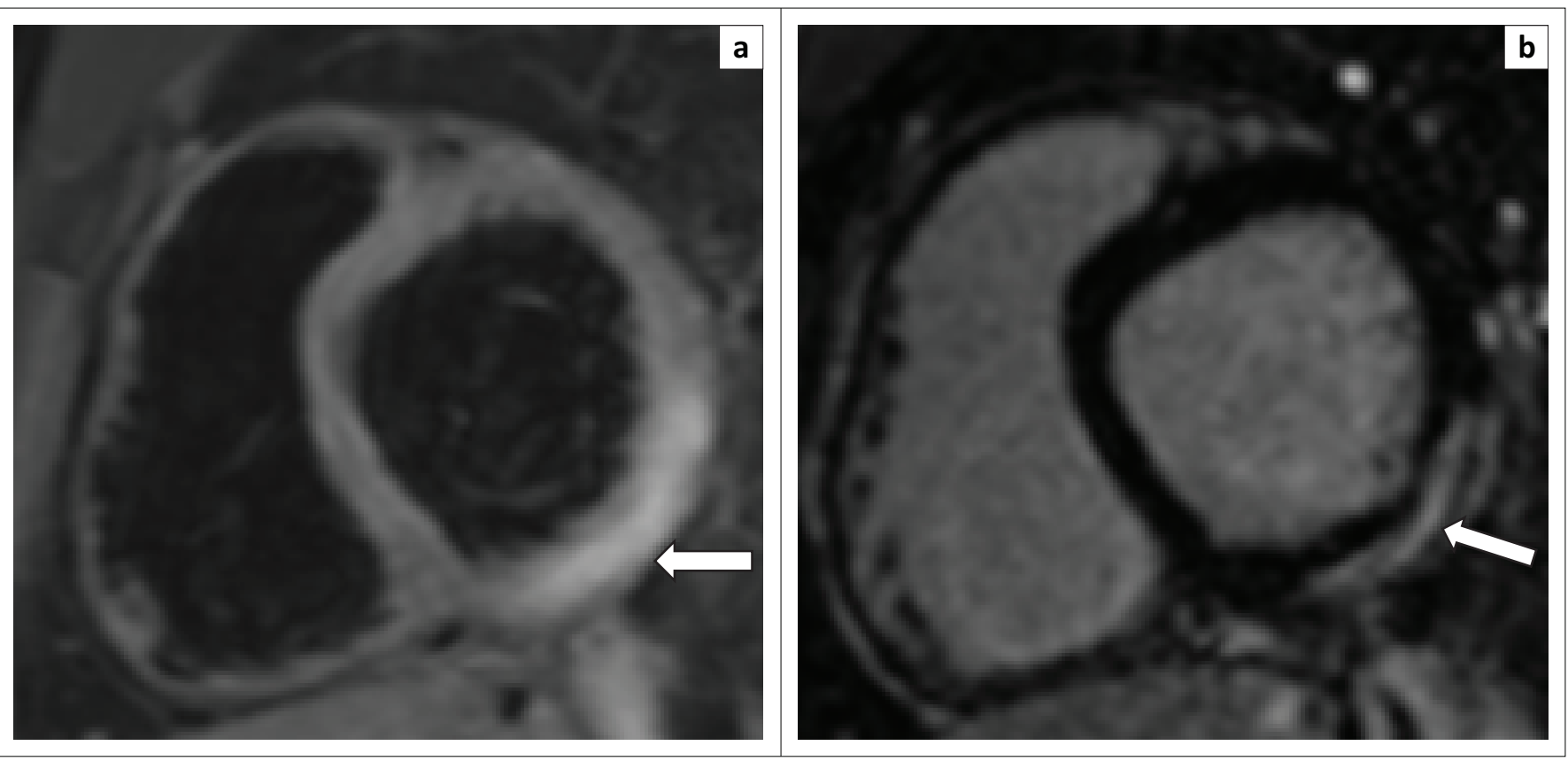

FIGURE 9: Myocarditis. T2W short-axis image (left) demonstrates LV inferolateral wall high signal and oedema (arrow). The equivalent late gadolinium image (right) demonstrates epicardial enhancement in the same region (arrow).

\section{Takotsubo cardiomyopathy}

Takotsubo (also called stress cardiomyopathy or catecholamineinduced cardiomyopathy) is typically seen in postmenopausal women in whom emotional stress may result in a clinical picture similar to acute myocardial infarction with anterior lead ECG changes. However, many patients do not report a clear stressor and this condition has been diagnosed in men. The rapidly reversible LV systolic dysfunction is caused by a temporary stunning of the apical to mid LV segments with apical ballooning, resulting in a dynamic obstruction. ${ }^{1}$

Myocardial oedema is evident as T2-weighted hyperintensity in a focal pattern, matching the wall motion abnormality and related to the severity of the systolic dysfunction. ${ }^{43}$ Lack of LGE is typical, and distinguishes it from myocarditis and infarction, but there may be mild patchy LGE composite with the myocardial oedema. ${ }^{44}$ The RV is involved in $25 \%$ of cases and complete resolution is typically at 4 weeks. ${ }^{1}$ Figure 10 demonstrates the representative imaging findings of takotsubo cardiomyopathy.

\section{Systemic sclerosis}

Systemic sclerosis is a connective tissue disorder affecting mainly the skin but also involving the lungs, kidneys and heart. ${ }^{45}$ Myocardial fibrosis is a common finding at autopsy studies. ${ }^{46}$ LGE manifests in a linear midmyocardial wall distribution at the basal and mid-ventricular levels. The development of clinical features indicates a poor prognosis, with a 5 - year survival rate of $30 \%{ }^{9}$

\section{Churg-Strauss}

Churg-Strauss is a rare small vessel syndrome with a poor prognosis. A subendocardial pattern of fibrosis in the absence of coronary artery disease is the typical pattern. ${ }^{47}$ Associated features are normal myocardial function, apical obliteration and thrombus formation. Elevated blood eosinophils are commonly found and imaging features overlap with hypereosinophillic cardiomyopathy.

\section{Rheumatoid arthritis}

Rheumatoid arthritis (RA) is a chronic autoimmune disease primarily involving the synovium, but extra-articular manifestations include cardiac involvement which can lead to a 10-year reduction in life span, especially in young women. CMR has been shown to detect diffuse and focal myocardial fibrosis as well as inflammation in RA patients. In addition, myocardial fibrosis and inflammation correlates with a C-reactive protein (CRP) score of disease activity as well as with systolic and diastolic strain patterns ${ }^{48}$ Myocardial fibrosis is not related with disease duration and T1 mapping can provide a new biomarker of disease activity in the heart, beyond that provided by the standard sequences such as short T1 inversion recovery (STIR) and LGE. ${ }^{48}$

\section{Phaeochromocytoma}

Cardiac involvement in phaeochromocytoma is frequent and often persistent, manifesting as myocarditis, global LV dysfunction, subclinical systolic and diastolic dysfunction as well as focal and diffuse fibrosis. The myocardial effects of phaeochromocytoma have been shown to be beyond that of hypertension; catecholamine-induced cardiac toxicity is the likely cause, leading to inflammation, myocyte necrosis and fibrosis. ${ }^{49}$

\section{Peripartum cardiomyopathy}

Peripartum cardiomyopathy is characterised by heart failure in the last trimester of pregnancy or first six post-partum 

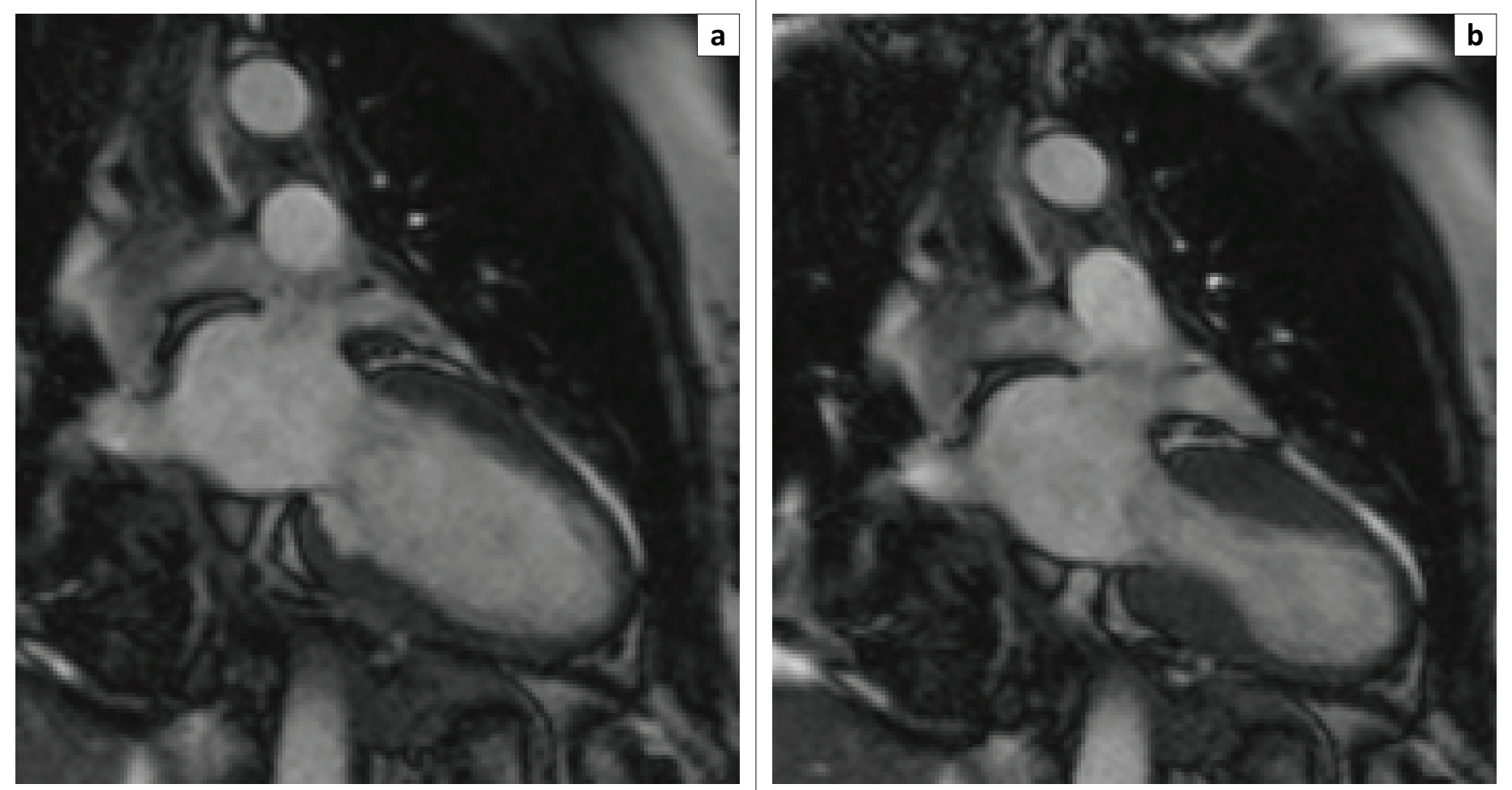

FIGURE 10: Takotsubo. Two-chamber cine b-SSFP at end-diastole (left) and end-systole (right) demonstrating a hypokinetic apex and mid LV, resulting in a Japanese 'octopus trap' appearance on the end-systole image.

months in previously healthy women. The cause is unknown and considered to be multifactorial. LGE is evident in the midmyocardium, mainly in the anterior and anterolateral segments. These features tend to regress over time as LV function improves. ${ }^{50}$

\section{Future perspectives and conclusion}

$\mathrm{CMR}$ is a unique non-invasive tool and currently integral part of cardiomyopathy work-up. Tissue characterisation remains its major strength, detecting fat, oedema, fibrosis, infiltration and infarction. Its excellent spatial resolution allows accurate evaluation of biventricular function and morphology. It is predicted that most patients with HF will eventually undergo CMR as part of diagnostic work-up and risk stratification. ${ }^{35}$ Its utility in the South African context is limited by the cost and availability of imaging expertise.

\section{Acknowledgements}

Dr Ntobeko Ntusi gratefully acknowledges support from the Harry Crossley Foundation, National Research Foundation and Medical Research Council of South Africa.

\section{Competing interests}

The authors declare that they have no financial or personal relationships which may have inappropriately influenced them in writing this article.

\section{Authors' contributions}

S.M. was the project leader. N.N. assisted with corrections and editing.

\section{References}

1. Parsai C, O'Hanlon R, Prasad S, Raad HM. Diagnostic and prognostic value of cardiovascular magnetic resonance imaging in non-ischaemic cardiomyopathies. J Cardiovasc Magn Reson. 2012;14:54-78. http://dx.doi.org/10.1186/1532-429X-14-54

2. Elliott $P$, Andersson B, Arbustini E, et al. Classification of the cardiomyopathies: $A$ position statement from the European Society of Cardiology Working Group on Myocardial and Pericardial Diseases. Eur Heart J. 2008;29:270-276. http://dx.doi org/10.1093/eurheartj/ehm342

3. Park HW, Cho $\mathrm{MH}$, Cho ZH. Real-value representation in inversion-recovery NMR imaging by use of a phase-correction method. Magn Reson Med. 1986:3:15-23. http://dx.doi.org/10.1002/mrm.1910030104

4. Moon JC, Messroghli DR, Kellman P, et al. Myocardial T1 mapping and extracellular volume quantification. J Cardiovasc Magn Reson. 2013;15:92. http://dx.doi.org/ $10.1186 / 1532-429 X-15-92$

5. Giri S, Chung YC, Merchant A, et al. T2 quantification for improved detection of myocardial edema. J Cardiovasc Magn Reson. 2009;11:56. http://dx.doi.org/ $10.1186 / 1532-429 X-11-56$

6. Alam $\mathrm{MH}, \mathrm{He} \mathrm{T}$, Auger $\mathrm{D}$, et al. Validation of $\mathrm{T} 2 *$ in-line analysis for tissue iron quantification at 1.5 T. J Cardiovasc Magn Reson. 2016;18:23. http://dx.doi. org/10.1186/s12968-016-0243-4

7. Cawley PJ, Maki JH, Otto CM. Cardiovascular magnetic resonance imaging for valvular heart disease: Technique and validation. Circulation. 2009;119:468-478. http://dx.doi.org/10.1161/CIRCULATIONAHA.107.742486

8. American College of Radiology. ACR-SPR practice parameter for the safe and optimal performance of fetal magnetic resonance imaging (MRI). Revised 2015 (Resolution 11) [homepage on the Internet]. [cited 2016 Apr] Available from: http://www.acr.org/ /media/CB384A65345F402083639E6756CE513F.pdf

9. O'Donnell DH, Abbara S, Chaithiraphan V, et al. Cardiac MR imaging of nonischemic cardiomyopathies: Imaging protocols and spectra of appearances. Radiology. 2012;262(1):402-422.

10. Pennell DJ, Sechtem UP, Higgins CB, et al. Clinical indications for cardiovascular magnetic resonance (CMR): Consensus Panel report. Eur Heart J. 2004;25:19401965. http://dx.doi.org/10.1016/j.ehj.2004.06.040

11. Kramer CM, Barkhausen J, Flamm SD, Kim RJ, Nagel E. Standardized cardiovascular magnetic resonance imaging (CMR) protocols, society for cardiovascular magnetic resonance: Board of trustees task force on standardized protocols. J Cardiovasc Magn Reson. 2008;10:35. http://dx.doi.org/10.1186/1532-429X-10-35

12. Maron BJ, Towbin JA, Thiene G, et al. Contemporary definitions and classification of the cardiomyopathies: An American Heart Association scientific statement from the council on clinical cardiology, heart failure and transplantation committee; quality of care and outcomes research and functional genomics and translational biology interdisciplinary working groups; And Council on epidemiology and prevention. Circulation. 2006;113:1807-1816. http://dx.doi org/10.1161/CIRCULATIONAHA.106.174287

13. McCrohon JA, Moon JC, Prasad SK, et al. Differentiation of heart failure related to dilated cardiomyopathy and coronary artery disease using gadolinium-enhanced 
cardiovascular magnetic resonance. Circulation. 2003;108:54-59. http://dx.doi. org/10.1161/01.CIR.0000078641.19365.4C

14. Lehrke $S$, Lossnitzer $D$, Schöb $M$, et al. Use of cardiovascular magnetic resonance for risk stratification in chronic heart failure: Prognostic value of late gadolinium enhancement in patients with non-ischaemic dilated cardiomyopathy. Heart 2011;97:e727-e732. http://dx.doi.org/10.1136/hrt.2010.205542

15. Assomul R, Prasad S, Lyne J, et al. Cardiovascular magnetic resonance, fibrosis and prognosis in dilated cardiomyopathy. J Am Coll Cardiol. 2006;48(10):1977-1985. http://dx.doi.org/10.1016/j.jacc.2006.07.049

16. Burke A, Mont E, Kutys R, Virmani R. Left ventricular noncompaction: A pathological study of 14 cases. Hum Pathol. 2005;36(4):403-411. http://dx.doi.org/10.1016/j. humpath.2005.02.004

17. Petersen SE, Selvanayagam JB, Wiesmann F, et al. Left ventricular non-compaction Insights from cardiovascular magnetic resonance imaging. J Am Coll Cardiol. 2005;46:101-105. http://dx.doi.org/10.1016/j.jacc.2005.03.045

18. Marcus FI, McKenna WJ, Sherrill D, et al. Diagnosis of arrhythmogenic right ventricular cardiomyopathy/dysplasia: Proposed modification of the Task Force Criteria. Eu Heart J. 2010;31:806-814. http://dx.doi.org/10.1093/eurheartj/ehq025

19. Sen-Chowdhry S, Prasad SK, Syrris P, et al. Cardiovascular magnetic resonance in arrhythmogenic right ventricular cardiomyopathy revisited: Comparison with task force criteria and genotype. J Am Coll Cardiol. 2006;48:2132-2140. http://dx.doi. force criteria and genotype. J Am
org/10.1016/j.jacc.2006.07.045

20. Sen-Chowdhry S, Syrris P, Prasad SK, et al. Left-dominant arrhythmogenic cardiomyopathy: An under-recognized clinical entity. J Am Coll Cardiol. 2008;52:2175-2187. http://dx.doi.org/10.1016/j.jacc.2008.09.019

21. Maron MS, Maron BJ, Harrigan C, et al. Hypertrophic cardiomyopathy phenotype revisited after 50 years with cardiovascular magnetic resonance. J Am Coll Cardiol. 2009;54:220-228. http://dx.doi.org/10.1016/j.jacc.2009.05.006

22. Maron MS, Finley JJ, Bos JM, et al. Prevalence, clinical significance, and natural history of left ventricular apical aneurysms in hypertrophic cardiomyopathy. Circulation. 2008;118:1541-1549. http://dx.doi.org/10.1161/CIRCULATIONAHA.108.781401

23. Rickers $C$, Wilke NM, Jerosch-Herold $M$, et al. Utility of cardiac magnetic resonance imaging in the diagnosis of hypertrophic cardiomyopathy. Circulation. 2005;112:855-861. http://dx.doi.org/10.1161/CIRCULATIONAHA.104.507723

24. Rubinshtein R, Glockner JF, Ommen SR, et al. Abstract 4187: Identification of late gadolinium enhancement by contrast-enhanced magnetic resonance Imaging as a major risk factor for sudden death in hypertrophic cardiomyopathy. Circulation. 2008;118:839S.

25. Maron BJ, Maron MS, Wigle ED, Braunwald E. The 50-year history, controversy, and clinical implications of left ventricular outflow tract obstruction in hypertrophic cardiomyopathy from idiopathic hypertrophic subaortic stenosis to hypertrophic cardiomyopathy: From idiopathic hypertrophic subaortic stenosis to hypertrophic cardiomyopathy. J Am Coll Cardiol. 2009;54:191-200. http://dx. doi.org/10.1016/j.jacc.2008.11.069

26. Selvanayagam JB, Hawkins PN, Paul B, Myerson SG, Neubauer S. Evaluation and management of the cardiac amyloidosis. J Am Coll Cardiol. 2007;50:2101-2110. http://dx.doi.org/10.1016/j.jacc.2007.08.028

27. Maceira AM, Joshi J, Prasad SK, et al. Cardiovascular magnetic resonance in cardiac amyloidosis. Circulation. 2005;111:186-193. http://dx.doi. org/10.1161/01.CIR.0000152819.97857.9D

28. Vogelsberg H, Mahrholdt H, Deluigi CC, et al. Cardiovascular magnetic resonance in clinically suspected cardiac amyloidosis: Noninvasive imaging compared to in clinically suspected cardiac amyloidosis: Noninvasive imaging compared to org/10.1016/j.jacc.2007.10.049

29. Maceira AM, Prasad SK, Hawkins PN, Roughton M, Pennell DJ. Cardiovascular magnetic resonance and prognosis in cardiac amyloidosis. J Cardiovasc Magn magnetic resonance and prognosis in cardiac amyloidosis. J

30. Clarke JT. Narrative review: Fabry disease. Ann Intern Med. 2007;146:425-433. http://dx.doi.org/10.7326/0003-4819-146-6-200703200-00007

31. Moon JC, Sheppard M, Reed E, Lee P, Elliott PM, Pennell DJ. The histological basis of late gadolinium enhancement cardiovascular magnetic resonance in a patient with Anderson-Fabry disease. J Cardiovasc Magn Reson. 2006;8:479-482. http:// dx.doi.org/10.1080/10976640600605002
32. Sado D, Maestrini V, Piechnik $\mathrm{S}$, et al. Noncontrast myocardial $T$ mapping using cardiovascular magnetic resonance for iron overload. J Magn 1 Reson Imaging. 2015;41:1501-1511. http://dx.doi.org/10.1002/jmri.24727

33. Sharma OP, Maheshwari A, Thaker K. Myocardial sarcoidosis. Chest. 1993;103: 253-258. http://dx.doi.org/10.1378/chest.103.1.253

34. Yared K, Johri AM, Soni AV. Cardiac sarcoidosis imitating arrythmogenic right ventricular fibrosis. Circulation. 2008;118:e113-e115. http://dx.doi.org/10.1161/ CIRCULATIONAHA.107.755215

35. Mahrholdt H, Wagner A, Judd RM, Sechtem U, Kim RJ. Delayed enhancement cardiovascular magnetic resonance assessment of nonischeamic cardiomyopathies. Eur Heart J. 2005;26:1461-1474. http://dx.doi.org/10.1093/ eurheartj/ehi258

36. Greulich S, Ferreira VM, Dall'Armellina ED, Mahrholdt H. Myocardial inflammationare we there yet? Curr Cardiovasc Imaging Rep. 2015;8:6. http://dx.doi org/10.1007/s12410-015-9320-6

37. Mahrholdt H, Goedecke C, Wagner A, et al. Cardiovascular magnetic resonance assessment of human myocarditis: A comparison to histology and molecular pathology. Circulation. 2004;109:1250-1258. http://dx.doi.org/10.1161/01. CIR.0000118493.13323.81

38. Abdel-Aty H, Boye P, Zagrosek A, et al. Diagnostic performance of cardiovascular magnetic resonance in patients with suspected acute myocarditis: Comparison of different approaches. J Am Coll Cardiol. 2005;45:1815-1822. http://dx.doi. org/10.1016/j.jacc.2004.11.069

39. Zagrosek $A$, Abdel-Aty $H$, Boye $P$, et al. Cardiac magnetic resonance monitors reversible and irreversible myocardial injury in myocarditis. JACC CardiovasC Imaging. 2009;2:131-138. http://dx.doi.org/10.1016/j.jcmg.2008.09.014

40. Karamitsos T, Francis J, Myerson S, Selvavayagam JB, Niebauer S. CMR in heart failure. J Am Coll Cardiol. 2009;54(15):1407-1424. http://dx.doi.org/10.1016/j. jacc.2009.04.094

41. De Cobelli F, Pieroni M, Esposito A, et al. Delayed gadolinium enhanced cardiac magnetic resonance in patients with chronic myocarditis presenting with heart failure or recurrent arrhythmias. J Am Coll Cardiol. 2006;47:1649-1654. http:// dx.doi.org/10.1016/j.jacc.2005.11.067

42. Ferreira VM, Piechnik S, Dall'Armellina ED, et al. T1 mapping in the diagnosis of acute myocarditis using CMR comparison to T2-weighted and late gadolinium enhancement. J Am Coll Cardiol Cardiovasc Imaging. 2013;6(10):1048-1058. http://dx.doi.org/10.1016/j.jcmg.2013.03.008

43. Eitel I, Behrendt F, Schindler K, et al. Differential diagnosis of suspected apical ballooning syndrome using contrast-enhanced magnetic resonance imaging. Eur Heart J. 2008;29:2651-2659. http://dx.doi.org/10.1093/eurheartj/ ehn433

44. Garg P, Greenwood J, Plein S. Multiparametric relaxometry by cardiac magnetic resonance imaging in Takotsubo cardiomyopathy. Eur Heart J Cardiovasc Imaging. 2015;16(10):1174. http://dx.doi.org/10.1093/ehjci/jev167

45. Varga JA, Trojanowska M. Fibrosis in systemic sclerosis. Rheum Dis Clin North Am. 2008;34(1):115-143. http://dx.doi.org/10.1016/j.rdc.2007.11.002

46. Kahan A, Allanore Y. Primary myocardial involvement in systemic sclerosis Rheumatology (Oxford). 2006;45(Suppl 4):iv14-iv17. http://dx.doi.org/10.1093/ rheumatology/kel312

47. Wassmuth R, Gobel U, Natusch A, et al. Cardiovascular magnetic resonance imaging detects cardiac involvement in Churg-Strauss syndrome. J Card Fail. 2008;14:856-860. http://dx.doi.org/10.1016/j.cardfail.2008.07.227

48. Ntusi NA, Piechnik SK, Francis JM, et al. Diffuse myocardial fibrosis and inflammation in rheumatoid arthritis: Insights from CMR T1 mapping. JACC Cardiovasc Imaging. 2015;8(5):526-536. http://dx.doi.org/10.1016/j.jcmg.2014. 12.025

49. Ferreira VM, Marcelino M, Piechnik SK, et al. Pheochromocytoma is characterized by catecholamine-mediated myocarditis, focal and diffuse myocardial fibrosis, and myocardial dysfunction. I Am Coll Cardiol 2016; 67(20):2364-2374. http://dx.doi.org/10.1016/j.jacc.2016.03.543

50. Kawano $\mathrm{H}$, Tsuneto $\mathrm{A}$, Koide $\mathrm{Y}$, et al. Magnetic resonance imaging in a patient with peripartum cardiomyopathy. Intern Med. 2008; 47(2):97-102. http://dx.doi. org/10.2169/internalmedicine.47.0316 